\title{
PREVENTION, MONITORING AND CONSERVATION FOR A SMART MANAGEMENT OF THE CULTURAL HERITAGE
}

\author{
ANTONELLA GUIDA \& VITO D. PORCARI \\ University of Basilicata, DiCEM-Matera, Italy
}

\begin{abstract}
Nowadays, the conservation of the cultural heritage requires the development of preventive protection strategies that should be increasingly innovative, effective, long-lasting, as well as economically sustainable. Although existing legislation provides for scheduled maintenance aimed at preventing deterioration and reducing operating costs, to date there are no decision support tools to assist in the periodic planning of the interventions to be executed. The aim of the research is to define the techniques and the innovative strategies of the maintenance process, focusing on the Smart capacity of such process. The research objectives are related to specific phases.

Fact-finding phase: Carry out actions and interventions for managing available resources and for implementing policies through the development of a technological platform for planning preventative maintenance operations.

The diagnostic phase: Carried out with the support of the heritage building information modelling methodology. Its aim is to define a specific degradation phenomenon in time and space, and to measure it in order to identify the appropriate steps to take for maintenance or restoration operations.

Participatory phase: The aim is the development of a multimedia platform, accessible to every type of user. This platform should allow the exchange of heterogeneous information, through the implementation of known systems for managing digital assets.

The final result of research will be the formulation of an information and communication technologies model that indicates the optimal maintenance frequency of a work of art based on its state of degradation (measured through indicators correlated with the nature of the material and the internal and external environmental conditions, continuously monitored), cost of maintenance and durability of the interventions performed.

An additional expected result will be the development of a participatory app, designed as a tool for promoting the accessibility and usability of the digital cultural content of an asset or of a cultural itinerary, and present it as a 'virtual tour', and for ultimately developing a broader 'digital culture' of the cultural heritage.
\end{abstract}

Keywords: cultural heritage, integrated recovery, scheduled maintenance, Smart technologies, durability.

\section{INTRODUCTION}

Today, the focus on the historic and modern architectural heritage is now centred on scientific interests specifically related to architectural technology, such as the recovery and promotion of assets, building design, and energy efficiency and saving.

The scientific interests of the research are oriented towards the use of traditional methodologies and technologies integrated with contemporary technical innovations in order to formulate the most appropriate recovery strategy and ensure an adequate state of conservation through a thorough knowledge of the architectural asset.

The importance of taking such an approach stems from the need to consider a recovery project not simply as the sum of individual skills from different sectors, but as a truly interdisciplinary exchange. Various specialists working in synergy can help promote a better understanding of the multiple problems encountered during the recovery and, at the same time, help identify and evaluate the suitability of the solutions proposed [1]. 
The historic architectural heritage and the current discussions surrounding the built environment have brought about a change in attitude towards the greater deterioration suffered by historic and the artistic heritage than was the case in the past. Such deterioration is caused essentially by the combined action of different factors: atmospheric pollution, climate change, biological contamination, anthropogenic degradation, and not least, the wrong approach taken during refurbishment work [2].

For these reasons, we intend to research and examine in depth those aspects and methods that are most appropriate to the improvement of the design methodology known as 'integrated conservation'. This relates to areas such as recovery, restoration, promotion and, above all, the preservation of our historic, architectural and urban heritage [3].

An important issue of growing interest is the conservation and preservation of the cultural heritage, which necessarily requires a 'preventive and scheduled strategy' for the execution of conservation and maintenance operations. This strategy is essential in order to guarantee the survival of the architectural asset in a consistent and coherent way.

\section{STATE OF THE ART}

For some time now, there has been a growing public awareness towards the preservation of the urban environment, and especially of what constitutes the historical heritage of a community. As in the case of human health, so for historic buildings, prevention is better than cure. In the case of the architectural heritage, prevention means a continuous and innovative monitoring [2].

The importance of using Smart monitoring methodologies is reinforced by the fact that all systems, including buildings, have a response time during which it is still possible to return them to the state they were in before being subjected to the stresses that disturbed them, without the system suffering permanent impairment. However, this response time must be shorter than the time the system was subjected to stress or threat; otherwise, the changes that occur tend to produce effects leading to degradation which will be difficult to reverse [4].

One of the initiatives which is investigating a possible solution to the response time issue is a project called 'MONSTER' ('Monitoraggio Strutturale di Edifici Storici con Tecnologie Wireless e Strumenti di Calcolo Innovativi', Structural Monitoring of Historic Buildings using Wireless Technologies and Innovative Computing Instruments), carried out by CNR-ISTI between 2014 and 2016. Two research laboratories were involved: Wireless Networks (led by Erina Ferro) and Mechanics of Materials and Structures (led by Cristina Padovani). During the project, the researchers studied and developed methodologies based on networks of lowcost wireless sensors for the monitoring of ancient masonry buildings. The end users of this project will be the institutions responsible for the preservation of the architectural heritage.

Furthermore, today, before starting any recovery intervention on historic buildings or buildings with a recognized cultural value, the tendency is for the various stakeholders to establish common ground before work starts, as complex design processes tend to cover every historical and technical aspect more often than before.

It is therefore important that the specifics of any recovery project be fine-tuned. This means defining in advance the scope, the mutual relationships, the autonomy and the complexity of the relationships between the various specialists involved in it.

The 'knowledge' of the architectural heritage is the driving factor behind any kind of evaluation or intervention. The observation of the building over its entire 'lifetime', from its conception and initial design to its present condition of abandonment or disuse, is what will determine the necessity for its recovery [4]. 
It is easy to recognize today the fundamental role played by the new multimedia tools in the shift towards enhanced models for the communication of knowledge, which make cultural offerings open and accessible to everyone. The digitalization of content is therefore the principle underpinning these new models. However, especially today, digital content needs to be structured and be easy to explore in order to reach a larger number of stakeholders.

If we consider the set of tasks and roles assumed by applied research within the knowledge transmission processes, a position of growing responsibility is being assigned to this research field within professional and institutional contexts. It is in fact thanks to the ideas and efforts of applied research that it is being assigned development tasks on various fronts including new processes and communication models, data generation platforms, digital knowledge sharing practices, development of improved technological devices for accessing information and usability of navigation tools on the web.

A significant organization that focuses on such innovative digitalization of content for the transferring and sharing of knowledge is the Network School of Digital Cultural Heritage, Arts and Humanities. It brings together over 50 organizations, including universities, research institutions, schools, technical colleges, cultural institutes, associations, public and private companies. The common aim is to create a 'multi-site campus' capable of developing an educational offering which is harmonized with the national system of education. The ultimate goal is to build a set of digital skills that are essential to engage with the challenges posed by an increasingly multifaceted and heterogeneous interaction with the Smart society, within the framework of a scalable model at European level.

Another interesting initiative promoted by the Ministry for Cultural Heritage and Activities and Tourism is the Memorandum of Understanding signed between the General Secretariat of Cultural Heritage and ASS.I.R.C.CO. (Associazione Italiana Recupero e Consolidamento Costruzioni, Italian Association for the Recovery and Consolidation of Buildings). The aim of this memorandum is the digitalization and dissemination of surveys and project data for the recovery of the architectural heritage, in order to implement or contribute towards the creation of a catalogued archive of the state of our cultural heritage and involve young graduate professionals in the required actions.

The sources for this archive are graduation degree and doctoral theses, final dissertations for post-graduate specialization and master courses in the disciplines of surveying, diagnostics, architectural history, restoration, consolidation and recovery of architectural heritage, structural engineering and seismic engineering. This archived material is a truly valuable resource, which triggers a virtuous circle for all involved parties. Students, the ministry, universities, associations and ultimately the general public (the end users) will benefit greatly from such a digital asset [3].

Considering the technical-digital context alone, despite the widespread adoption of building information modelling (BIM) for the design and management of the life cycle of new buildings, very little research has been undertaken to explore the benefits of using the BIM methodology in the management of historic buildings and cultural landscapes. To this end, studies are in progress for the development of BIM platforms that incorporate both quantitative items (smart objects, performance data) and qualitative items (historic photographs, oral histories, music) [4] .

In addition, the three-dimensional (3D) models generated by this system use BIM software functionalities to provide a navigable timeline, which displays both material and immaterial changes to buildings that occurred in the past, as well as generating projections into the future. In this context, a wider and more innovative use of BIM for the documentation and conservation of the architectural heritage is being discussed. 


\section{CRITICALITY AND SOLUTIONS IN THE REFURBISHMENT PROJECT}

Today, the conservation of cultural heritage context is necessary to intervene with a preventive protection strategy that is increasingly innovative, effective, long-lasting as well as economically sustainable.

There are no decision support tools to assist in the periodic planning interventions to be carried out, even if the currently existing legislation provides for a scheduled maintenance aimed at preventing deterioration and reducing operating costs.

The aim of a recovery project should be the improvement of the overall behaviour of an architectural asset. This improvement is attainable through an indispensable 'knowledge' phase, which characterizes any restoration work and which encompasses all components and disciplines that define the architectural asset itself, allowing for an understanding of its essence and an appreciation of its qualities.

The purpose of the research is to define the techniques and innovative strategies in the maintenance process, focusing on the Smart capacity of this process.

The research objectives are linked to specific phases:

The fact-finding phase, which is preparatory to any recovery process, will help define the characteristics, issues and potential of each of the successive phases that result from a recovery project based on SMART materials, innovative technologies and methodologies.

The immediate next step should be the drafting and planning of a maintenance programme which will cover the management, analysis and verification of all data gathered. This is in order to ensure a complete collection of information on both the artefact being examined (from its construction and including all its subsequent transformative and maintenance phases), and the environmental context in which it is found.

In this context, and considering the current state of the art, one of our main scientific challenges is the development of new methodologies for the analysis and integration of data acquired from cross-platform sensors with different spatial and temporal resolutions. Such methodologies will allow experts from multiple disciplines to study highly complex environmental processes in innovative ways [5].

This original and innovative approach will be used to meet the requirements and needs of the community, of engineers, of employees and of end users in order to manage and promote natural resources, with the ultimate goal of creating a participatory awareness towards recovery and restoration within the community. This participatory philosophy, typical of the architectural domain, aims at the intelligent sharing of knowledge among engineers, businesses, and central and local government. As a result, data and details of the different phases of a project are made accessible to everyday users or to specialist researchers. The intelligent sharing of a project can be achieved through innovations in the existing and future monitoring instruments and in land management, through the integration of data obtained by different technologies (information and communication technologies [ICT], sensors, remote sensing) using the web and the new possibilities offered by navigation technologies and satellite telecommunications, from cloud computing to web sensors [6].

Today, research focuses its attention on building information modelling (BIM) application procedures applied to knowledge management, documentation and the planning of interventions on buildings. BIM is a methodology, which is still being optimized, for the organization of information archived in parametric objects and updated in real time through monitoring activities. Research work is planned with the aim of integrating the full incremental knowledge process within the BIM platform using the HBIM (historic BIM) approach for the management and recovery of the cultural heritage. Such an innovative knowledge management tool is 
implemented and continuously updated through the collection, cataloguing and digitalization of the precious and unique documentation found in peripheral bodies of the Ministry for Cultural Heritage and Activities such as the State Archives. Given its breadth of vision and the number of areas involved, we can foresee an expansion and a consolidation of its relationships with all the fundamental disciplines, such as archaeology, hydrology, geology and others, all of which have a common interest in the recovery, promotion, security of the heritage and preventing risk to it.

\section{OBJECTIVES AND METHODOLOGIES}

Considering the various topics and areas of interest to be covered, we can identify different phases and related objectives.

1. Fact-finding phase. Implement actions and interventions for the management of the resources available and for the implementation of policies through the development of a technology platform for scheduling preventive maintenance. Acquire as complete a view as possible of the state of conservation of the asset. This step is crucial for executing maintenance work correctly and preventively, for saving on costs (due to less risk of damage to the asset), for modelling the information and for identifying objective metrics that describe the degradation rate of the assets subject to observation and intervention. The specific methodology for this objectives is building a user-friendly database through the collection, classification and digitalization of documentation held in the State Archives and in the local authority archives (such as Superintendencies), as well as project data coming from freelance professionals. Such information, combined with the data constantly being monitored, will allow the fine-tuning of the BIM simulation models on which the design choices of restoration or maintenance operations will be configured.

2. Diagnostic phase. The diagnostic phase is realized with the support of HBIM methodology. Its purpose is to define specific degradation phenomena in time and space, and measure it in order to identify the necessary steps to keeping or restoring. The final goal is to define areas suitable for investigation in order to fully exploit the capabilities offered by BIM for existing buildings and enhance the traditional process of the acquisition of knowledge through ICT.

The methodology is the combination of monitoring systems and BIM methodology realizes the concept of an instrumented building, digitized by a 3D model capable of automating the activity recording devices triggered by the sensors. Some examples of monitoring applications integrated in the BIM platform are real-time acquisition of performance data related to plants in order to detect malfunctions or failures; on-site measurement under variable boundary conditions (weather, users' needs, technical system to be controlled) and amplitude measurements of spreading cracks or of ongoing subsidence through strain gauges (structural monitoring).

3. Participatory phase. The objective is to develop a multimedia platform, accessible to every type of user (allowing for an area reserved for professionals and engineers). This platform should enable the exchange of heterogeneous information, through the implementation of known systems (such as Google Earth) for managing digital assets from a socioeconomic perspective and in order to promote and disseminate culture and art, also in conjunction with the tourism industry.

Finally, the methodology for this phase. The participatory approach to such an innovative design process is guaranteed by the deployment of a unified data model, based on an ontological structure specifically dedicated to engineers and professionals. Such approach is crucial 
in order to execute conservation work correctly and preventively. We will develop a prototype application for smartphones and tablets, using Google Earth as geo-reference base. Such an application will include advanced information generated to dynamically design a system that would allow users to get to know and tour sites through interactive, photorealistic, 3D representations, navigable in real time, therefore giving them access to places where the 'Design for All' principles could not be adhered to.

\section{CASE STUDIES}

The activities presented in this article is part of a more general research under way, It aims to identify, detect, classify and interpret the historical and architectural heritage with the aim of coordinated and controlled management of degradation and conservation processes. Such activities, extended to the entire national heritage, will initially be validated in the city of Matera, a World Heritage Site since 1993 and European Capital of Culture 2019, through three ecclesiastical architectures used as pilot cases to achieve and implement a protocol and a unified methodology to preserve and maintain the value of the cultural heritage

The three churches are the church of 'San Francesco d'Assisi', the cave church of 'San Pietro Barisano' and the cave church of 'Santa Lucia alle Malve'; the choice has been conducted by the will to intervene and interpret the two different but contextual nature of traditional architecture in a similar context: built and excavated.

In fact, the church of San Francesco d'Assisi (Fig. 1), located just off the main piazza Vittorio Veneto, in a part of the urban perimeter defined as 'the city of the Plan' who consider the built city. The architecture presents a broad and regular baroque façade, dating back to the 18th century, although the original 200 , as it was modified several times until reaching its current appearance.

The cave church of Santa Lucia alle Malve (Fig. 2), however, is the first female monastic settlement of the Benedictine Order, dating back to the 8th century, and the most important

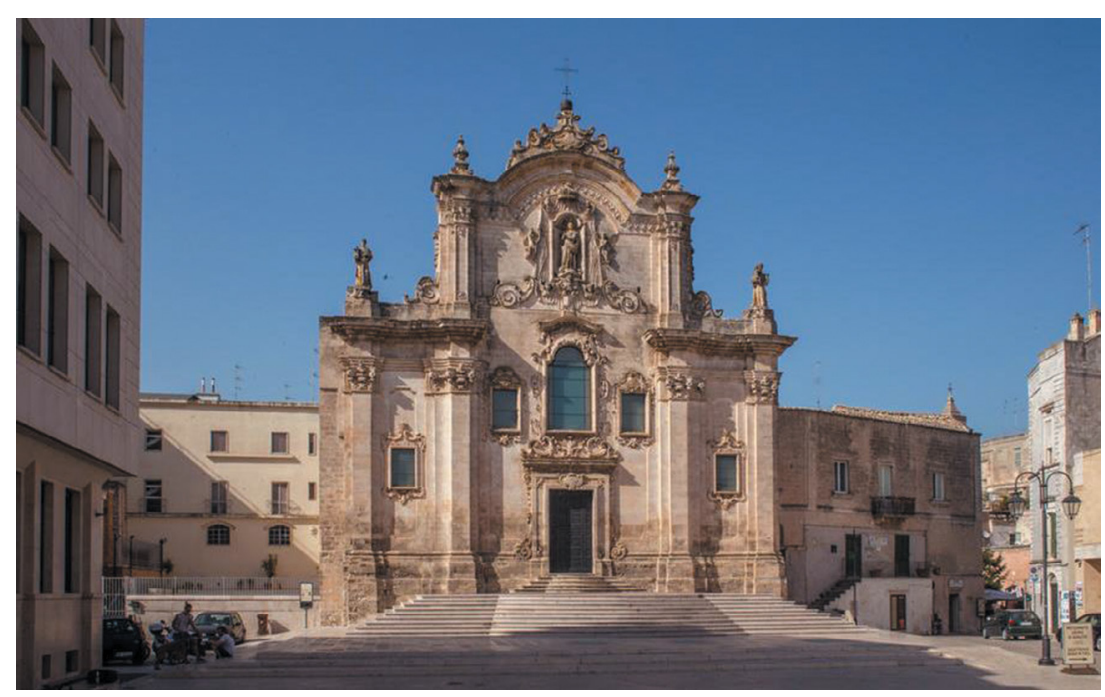

Figure 1: Church of San Francesco d'Assisi, Matera, Italia.

(visitmatera.it) 


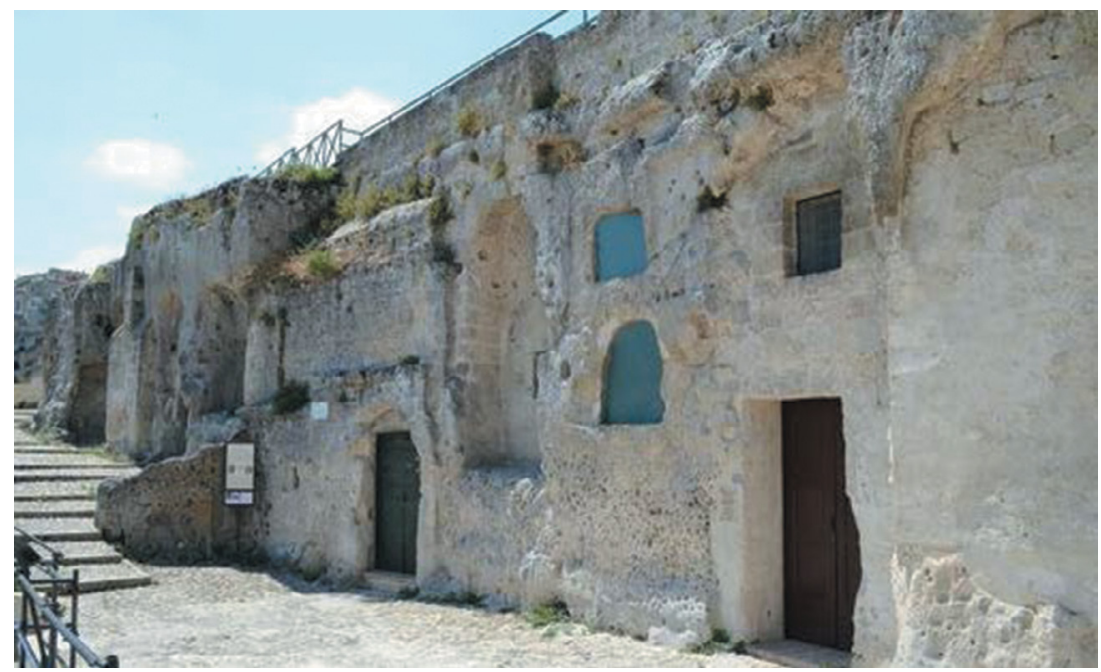

Figure 2: Church of Santa Lucia alle Malve, Sasso Caveoso, Matera, Italia. (tripadvisor.it)

in the history of the city of Matera being one of the most important evidences of excavated architecture, in the Sasso Caveoso.

The external façade of the former monastery complex stretches along the rocky wall with a number of accesses that enter in as many internal cavities. The interior of the church has three naves separated by columns. The right aisle is still open for worship, and again here today we celebrate the anniversary of the saint on December 13.

The other two aisles had been used as a dwelling. The aisles are richly adorned with frescoes dating back to the 12th century, including the Madonna del latte and San Michele Arcangelo.

Above the church is located a necropolis with tombs in the rock.

Finally, the church of San Pietro Barisano (Fig. 3), is a perfect example of the typical architectural structure of the Sassi: have a façade built in the tufo, but the interior is almost completely excavated. The church dates back to the year 1000 and was known as San Pietro in Veteribus, but the façade that presents us today dates from 1755, as it shows an inscription on it, when the church was renovated and partly modified.

The interior consists of three naves divided by columns carved supporting round arches, and has six altars that also dug into the tufo. The main altar is of 18th century and is made of gilded wood.

We have started the survey campaign with 3D scanning technique using laser scanner so we can get the autonomous acquisition millions of 3D points in a very short time and with much higher quality than the classical relief tools; also, we performed punctual thermo-visions through thermal imaging camera which non-destructive means of diagnostic techniques to check and detect moisture phenomena, hidden architectural elements, identify gaps in the plaster and identify the structural part of the building.

Below, there are certain findings made on two of the sites concerned: the church of Santa Lucia alle Malve and the church of San Pietro Barisano (Figs. 4 and 5). 


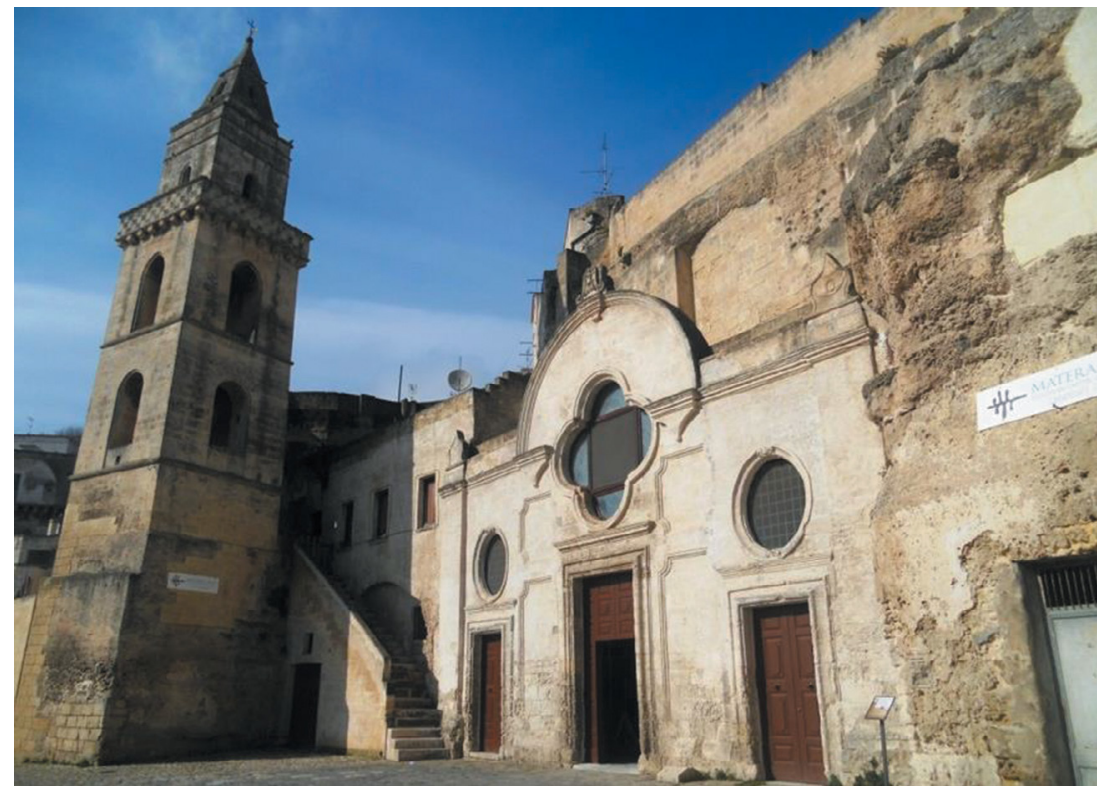

Figure 3: Church of San Pietro Barisano, Sasso Barisano, Matera, Italia. (guida-matera.it)

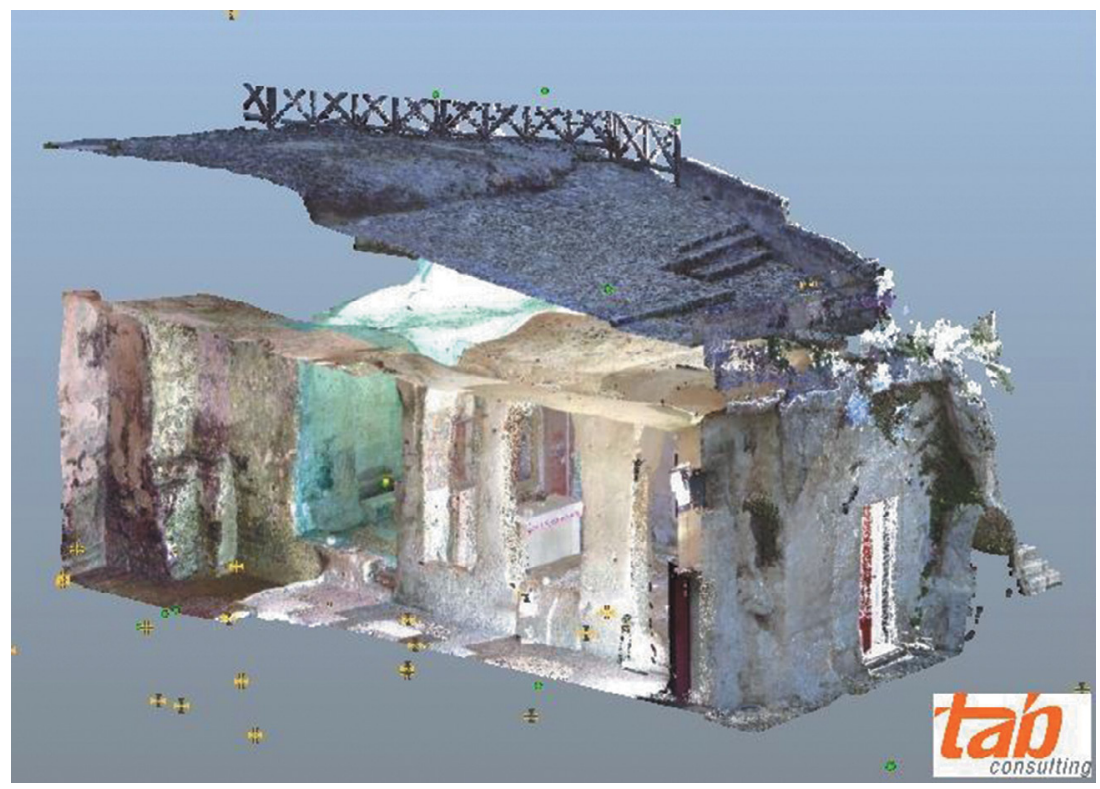

Figure 4: Section of the complex point cloud of the church of Santa Lucia alle Malve. 


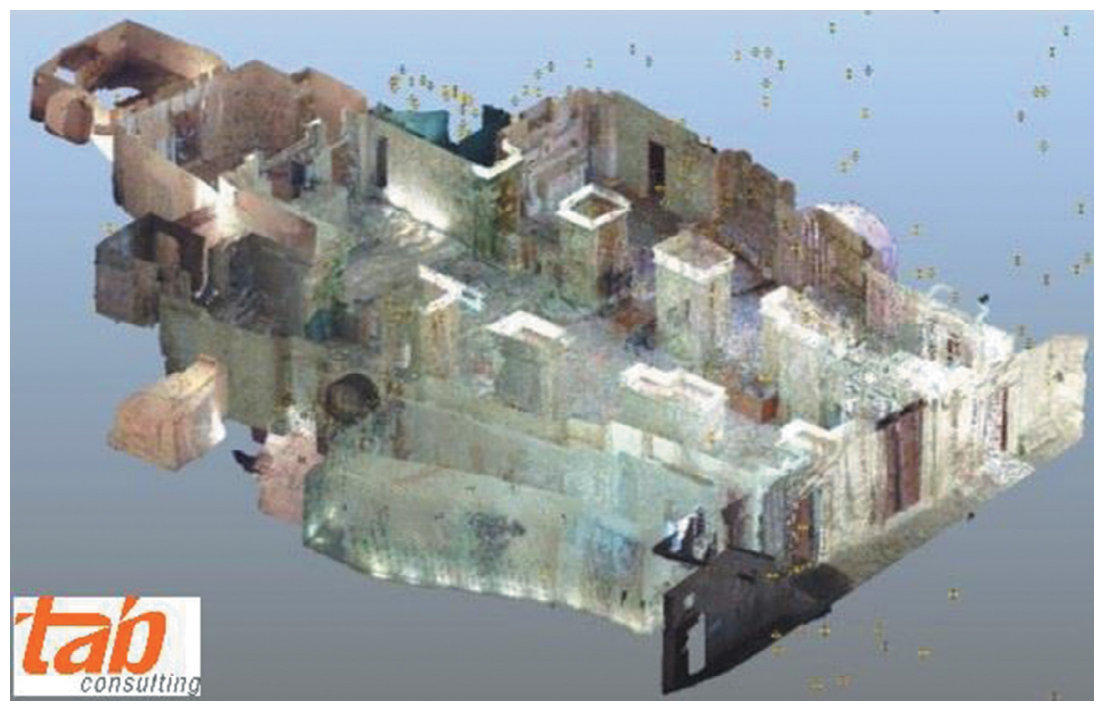

Figure 5: Axonometric section of the complex point cloud of the church of San Pietro Barisano.

\section{RESULTS AND CONCLUSIONS}

The final result of the research will be the formulation of an ICT model that indicates the optimum maintenance frequency of an architecture according to its state of degradation (measured by means of indicators related to the nature of the material and the internal and external environmental conditions, continuously monitored), maintenance costs and durability of the interventions.

A further result of my research efforts will be therefore the creation of a technological platform composed of a network of sensors, on-site and off-site measuring instruments, and a data storage and processing system for planning maintenance operations and for managing interventions. In addition, we intend to develop a unified procedure based on 'Adaptable Design' guidelines that integrates, both in form and technique, the above measuring and monitoring instruments (sensors and so on) within the architectural asset being monitored.

Another expected result will be the development of a participatory application, designed as a tool to promote accessibility and usability of digital cultural content of an asset or a cultural route and present it as a 'virtual tour' and for the development of a 'digital culture' of the cultural heritage, in order to keep contemporary users up-to-date, as well as to prepare and support future generations.

Such an application is therefore aimed at a diverse target audience. Local ministerial authorities could use it as a tool for collecting significant data that are useful for managing emergencies and for planning maintenance operations. Researchers could use this app as an innovative knowledge tool that provides input for the above-mentioned data collection carried out by the ministerial authorities (output).

The application could also be used in cases of destruction and total loss of architectural and monumental assets caused by natural hazards that sadly occur all too frequently, to allow a fast and complete survey for their rebuilding or as a 'memory' of the destroyed assets. At the same time, design engineers could also use this application as a base tool for planning their work, as 
well as the general public, who could use it simply as an aid for everyday cultural tourism, for visiting and exploring sites in real time through interactive, photorealistic, 3D representations.

Finally, it can be said as the most appropriate strategy must tend to an ever more effective risk prevention also generated by natural events such as the earthquake, in a preventive perspective than the usual intervention of failure, then go from the emergency restoration to ordinary conservation (maintenance); therefore, the activity undertaken aims to define and seek a practical solution to this complex and sensitive issue, through an effective, non-existent today, through which to identify and plan the interventions of high priority using digitization and current instrumentation to make each building Smart.

So, by intervening using this method on every single building structure, the Smart acceptation can be representative of entire towns, and Matera is a candidate to be.

\section{ACKNOWLEDGMENTS}

The authors would like to mention and acknowledge the 'Tab Consulting S.r.l.' in Potenza (Italy) for collaboration in the survey campaign.

\section{REFERENCES}

[1] Guida, A., Pagliuca, A. \& Tranquillino Minerva, A., A 'Non-Invasive' technique for qualifying the reinforced concrete structure, Hindawi Publishing Corporation, International Journal of Geophysics, 2012, Article ID (659612), p. 9, 2012. DOI: $10.1155 / 2012 / 659612$

[2] Guida, A. \& Pagliuca, A., Static requirement and type's complexity in the diagnostic phase. Journal of Civil Engineering and Architecture, 6, (4), April 2012 (serial Number 53), ISSN 1934-7359, pp. 489-495, USA, David Publishing Company, 2012. DOI: $10.17265 / 1934-7359 / 2012.04 .009$

[3] Guida, A., Dimitrijevic, B. \& Pagliuca, A., Reinforced Concrete Condition Assessment in Architectural Heritage. The Lion Chambers: Glasgow, UK; the Theatre E. Duni: Matera, Italy), Ed. CUES - Italy, 2011.

[4] Eastman, C., Teicholz, P., Sacks, R. \& Liston, K., BIM Handbook: A Guide to Building Information Modeling for Owners, Managers, Designers, Engineers and Contractors, 2nd edn, New Jersey: Wiley, Hoboken, 2011.

[5] Guida, A., Masini, N., Persico, R. \& Pagliuca A., A multifrequency and multisensor approach for the study and the restoration of monuments: The case of the Cathedral of Matera, in 'Advances in Geosciences (ADGEO)', Managing Editor: Arne Richter. International Journal of Advanced Geosciences, 18(1-6), 2008. DOI: 10.5194/ adgeo-19-17-2008

[6] The Federal Government, The new High-Tech Strategy Innovations for Germany, BMB, Germany, 2014.

\section{BIBLIOGRAPHY}

Di Biase, C., Il restauro e i monumenti. Materiali per la storia del restauro, CLUP: Milano, 2003.

Jokilheto J., 1999. A History of Architectural Conservation, Elsevier Butterworth-Heinemann: Oxford, 1999. 